\title{
Fluid-structure coupling analysis and simulation of a slender structure
}

\author{
A. Vasallo, A. Foces \& A. Lorenzana \\ Department of Architectural Constructions, Ground Engineering and \\ Mechanics of Continuous Means and Theory of Structures, \\ Valladolid University, Spain
}

\begin{abstract}
The analysis of the interaction between the flow and the fluid and an object represents a classical challenge for modern numerical techniques. Current work will concentrate on the case of bluff-body cross-sections featuring sharp corners and a clear predominance of the shape resistance over the friction resistance. For this category of structures the dynamic behaviour of the overall coupled system plays a very important role. In this work, the interest focuses on the behaviour of a beam structure subjected to a flow orthogonal to the beam axis. Under this assumption the flow at two points, at reasonable distance, will present little correlation, which allows to consider the flow at one point as uncoupled from the flow at other points along the same beam. This suggests the possibility of "slicing" the fluid domain in a number of independent two-dimensional planes on each of which the problem can be solved separately. Conceptually the solution on each slice will provide a force density acting on the beam, obtained by integrating the pressure of the fluid over the corresponding cross-section. This can be interpreted as a time-varying distributed load over the beam. The aeroelastic analysis of a slender beam is performed coupling a Navier Stokes Solver with the structural model. In the analysis of the structure is used a monodimensional structural model applicable to thin-walled composite beams, which can have either an open or closed profile with either a single- or multiplecell section. However, in the application example presented here, a steel chimney 90 meters tall is analyzed.
\end{abstract}

Keywords: coupled problem, beam, thin-walled, one-dimensional model. 


\section{Introduction}

Over the last 40 years the Finite Element Method (FEM) experimented an exponential growth which led to the definition of a set of reliable computational techniques for many different problems. This maturity, together with the increasing availability of powerful computational resources gave rise in relatively recent times to an increasing interest in coupled problems.

The recent works $[1,2]$, provide an interesting introduction to the subject together with a review of modern analytical techniques for the assessment of stability and accuracy of different staggering techniques. The case treated is mostly that of structure-structure interaction which represents a limit case of coupled system; however some results are general and can be extended to a broader range of fields.

Particularly in the case of interaction between flexible structures and incompressible fluids (model led using the Navier-Stokes equations), which is the case of interest for us; the assumption of linearity for the fluid simply does not hold because of the mathematical structure of the problem. Given the non linearity of the problem, the two coupled fields involve the definition of a nonlinear system of equations which could be linearized using the Newton-Raphson method. As expected, the off-diagonal terms of the tangent stiffness matrix express the dependency of one field on the variations of the second. It can be observed how because of the mathematical structure of the fluid problem any perturbation on the boundary propagates very quickly (at the limit instantaneously for truly incompressible fluids) to the whole fluid domain. Even, if the linearization of the fluid field were possible in theory, it would not be usually performed as it is often preferred to rely on fixed point type iterations to solve the non linearities. It follows that the calculation of the jacobian would involve a complete redefinition of the fluid strategy which is a nightmare from the point of view of software modularity.

Finally, it is generally difficult to find a satisfactory test system particularly as the requirement of "physical stability" is often not easy to meet in the practice which endangers the last assumption.

Two main approaches exist to solve the impasse. One "purely algebraic" focused on the techniques to solve iteratively the system of equations. The second approach focused on "explicit" coupling techniques where convergence to the coupled solution is obtained by adjustment of the predictors and of the data transferred between the domains.

Given the absence of a common mathematical formulation for the two coupled fields, the investigation of the stability properties of the different methods is in this case extremely challenging. A 1-D case is investigated by Larroutorou et al [3] (for a compressible case) while Farhat and Piperno [4] a simple test is advocated to assess the prophension of a given method to instability. Even, if the proposed method has no general mathematical validity it was shown to discriminate successfully stable methods from unstable techniques. The techniques proposed were tested on large aeronautical examples (e.g. Brown 
et $a l[5])$ and provided satisfactory results in describing experimental flutter envelopes on real aircrafts.

Relevant contributions to the subject (for the incompressible case) can be found in Del Pin et al [6], where the interaction problem is naturally dealt with by using an innovative lagrangian formulation for the fluid flow which allows to deal naturally with arbitrarily large mesh displacements. The method described is based on an extremely fast mesh regeneration technique joint with a fractional step formulation for the fluid domain.

Finally, Space-Time Finite Element based approaches can be found in Hubner et al [7] and Dinkler et al [8] with reference to both civil and aeronautical applications. Examples of applications to large scale cases of flexible civil engineering structures can be found in Gluck et al [9] and Halfmann et al [10].

On the other hand, the structural model used to solve the structure has been developed by de same authors Foces et al [11]. This model was developed for the analysis of thin-walled composite beams. Each wall is made of orthotropic layers bonded together to form a laminate that can be anisotropic. A beam element was got which is suitable for the simulation of both open-section or closed-section beams of arbitrary section shape with arbitrary layup. Nevertheless, this structural model is applicable to isotropic beams, since these are a particular case of the previous ones.

\section{Structural model}

In general lines, an analytical monodimensional model was developed by Foces et al [11] to describe the behaviour of a thin-walled composite beam in bending and torsion.

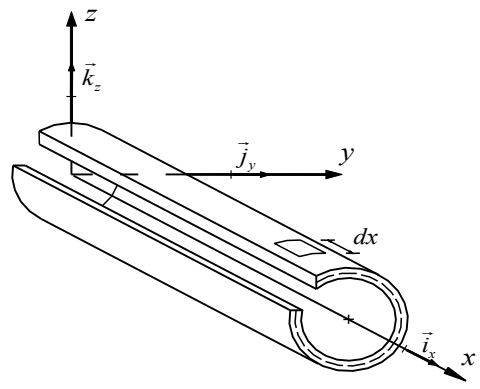

(a)

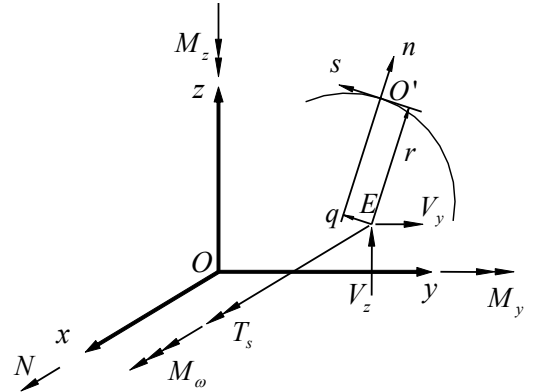

(b)

Figure 1: (a) Coordinate systems. (b) Generalized beam forces and moments.

The methodology proposed uses a Love-Kirchoff shell model to relate the stresses and strains in the shell. In this relation are introduced the generalized beam deformations corresponding to the Navier-Bernoulli and Vlasov models through geometric considerations. By integrating the stresses on the cross section, a relation between the generalized stresses and strains of the beam in the form of a symmetric $5 \times 5$ cross-sectional stiffness matrix $\boldsymbol{K}$, is got. 


$$
\boldsymbol{F}=\boldsymbol{K} \cdot \boldsymbol{q}
$$

where

$$
\begin{aligned}
\boldsymbol{F}^{T} & =\left[\begin{array}{lllll}
N & M_{y} & M_{z} & T_{s} & M_{\omega}
\end{array}\right] \\
\boldsymbol{q}^{T} & =\left[\begin{array}{lllll}
U_{, x} & \beta_{y, x} & \beta_{z, x} & \phi_{, x} & \phi_{, x x}
\end{array}\right]
\end{aligned}
$$

where $N$ is axial force, $M_{y}$ and $M_{z}$ are bending moments about the $y$ and $z$ directions respectively, $V_{y}$ and $V_{z}$ are transverse shear forces, $T_{s}$ is the St. Venant twisting moment, $M_{\omega}$ is a Vlasov bimoment, $U$ is the displacement of the beam along $x$-axis, $\beta_{y}$ and $\beta_{z}$ are rotations of the cross-section beam about the $y$ and $z$ axes respectively, and $\phi$ is the twist of each section around the $x$-axis, fig. 1 .

Finally, a beam element can be obtained applying the Principle of Virtual Works

$$
\int_{V^{(e)}} \delta \varepsilon \cdot \sigma \cdot d V=\delta q^{(e)} \cdot f^{(e)}
$$

Two different types of interpolation functions are introduced to describe the behaviour of the beam. For the axial displacement $U$, a two-node Lagrangian representation is used. For the cross-sectional rotations $\beta_{y}$ and $\beta_{z}$, and for twist deformation $\phi$ and its derivative $\phi_{, x}$, a two-node Hermite shape function is employed to satisfy the $C^{l}$ continuity at each extremity of an element. These yield a total of 14 degrees of freedom for each finite element that can describe extension, bending, and torsion. Introducing this finite element representation into the energy expression, eqn (3), we obtain the following set of finite element beam equations

$$
\boldsymbol{F}_{g}=\boldsymbol{K} \cdot \boldsymbol{q}_{g}
$$

where $\boldsymbol{K}$ and $\boldsymbol{F}_{g}$ are the finite element system of stiffness matrix and load vector respectively, and $\boldsymbol{q}_{g}$ is the generalized displacement vector for the beam.

\section{Fluid-structure coupling}

Multidisciplinary problems involving Fluid-Structure Interaction (FSI) are common in engineering design. In this case, a monodimensional structural model has to be coupled to the fluid flow. In order to perform the coupling it is therefore necessary to associate to the beam its three-dimensional "skin". Under the usual assumptions, the motion of the beam axis describes univocally the motion of its cross section, which can be imagined as a rigid body which follows the translation and the rotation of the beam axis.

This allows associating to each point of our monodimensional beam, assumed to be oriented as the $O z$-axis, a section of finite dimensions lying in the $x y$-plane. Given the hypothesis of small-strains, the motion of the cross section outside of the $x y$-plane can be neglected. This is not consistent with the kinematics hypothesis on the beam motion but can be accepted, as a very good approximation, for the only purpose of describing the motion of our beam imagined as a three dimensional object. The next step is the choice of the fluid model to be used for the structural solution.

Current work will concentrate on the case of bluff-body cross-sections featuring sharp corners and a clear predominance of the shape resistance over the 
friction resistance. For this category of structures the dynamic behaviour of the overall coupled system plays a very important role. A time-accurate viscous flow solver is thus needed. Even if the features of the problem make possible the use of a rather "coarse" mesh, see for example Rossi [12], it can be immediately verified that the number of elements needed for a complete 3-D simulation makes it unviable for any realistic beam length.

In many cases however the interest focuses on the behaviour of a beam subjected to a flow orthogonal to the beam axis. Under this assumption the flow at two points (at reasonable distance) will present little correlation, which allows to consider the flow at one point as uncoupled from the flow at other points along the same beam. This suggests the possibility of "slicing" the fluid domain in a number of independent two-dimensional planes on each of which the problem can be solved separately.

Conceptually the solution on each slice will provide a force density acting on the beam, obtained by integrating the pressure of the fluid over the corresponding cross-section. This can be interpreted as a time-varying distributed load over the beam. The deformation of the beam in turn will provide a relation between the motions of the different sections which should bring the simulation closer to the equivalent complete three dimensional simulation.

As a comment, we would like to propose that the incoming turbulence can be taken in account by generating a spatially correlated velocity field, see for example Rossi et al [13], to be applied as inflow velocity between the various "fluid slices" while performing a FSI analysis in the vicinity of the beam. This could also allow investigating the importance of the buffeting action in initializing the flutter of a given structure, e.g. Lazzari [14], or simulating the interaction of different cables subjected to different levels of stress.

Before proceeding further, it is interesting to make a philosophic consideration on the nature of the coupling. The beam formulation makes use of the small strain hypothesis which implies that the reference and deformed configuration are considered to coalesce at least in writing the equilibrium. The motion of the cross-section on the other hand is obtained "exactly" once given the motion of the corresponding axis, without taking advantage of the smallstrain hypothesis. This implies that the loads acting on the structure will be allowed to depend in a non-linear way from the motion of the beam. This feature may become important for the cases in which the model moves "at the limits" of the small strain formulation.

To complete the discussion we need to choose a suitable coupling algorithm. The problems of interest falls in the realm of aeroelasticity and will be often characterized by large Reynolds numbers and flows featuring sharp separations at the corners of the section. Loose coupling procedures are known to perform very efficiently for such category of problems, see for example Farhat and Piperno [4]. The choice in our case will be the Fractional-Step approach described in Rossi [12] which assumes the form

- Solve the structure using the loads (pressure) at time.

- Move the mesh according to the structural motion.

- Solve the fluid. 
- Solve the structural problem applying the newly calculated pressure.

The properties of this algorithm are analysed in Rossi [12]. In detail, the structural solution assumes, for the case of our beam, the form

- Integrate the fluid force on the bridge section.

- Solve the dynamic problem.

- Move the section accordingly to the calculated motion of the beam.

- Assign a velocity to the points of the section consistently with the mesh motion scheme chosen (velocity $=$ mesh_velocity (displacement)).

The fluid solver used is a Fractional-Step type procedure featuring a second order pressure splitting as described in Codina [15]. The solver allows an arbitrary Lagrangian-Eulerian (ALE) description of the fluid domain allowing dealing simply with the deformation of the fluid medium. The stability properties for the scheme are described in detail in Badia [16]. Stabilization of convection and diffusion is obtained using the Orthogonal Sub Scales (OSS) approach as proposed in Codina [17]. Quasi-static subscales are assumed. No turbulence model is included in the simulation. The structure is integrated using a standard second-order accurate Newmark scheme without viscous damping.

\section{Example}

The aeroelastic analysis of slender beam structures represents a rather new area of study. To our knowledge no experimental or numerical results are available on the subject, the rationale followed here is therefore to analyse a simple setup in order to propose a benchmark. The analysis is performed inside the code Kratos, a general purpose multiphysic code which was already validated in Rossi [12] in application to problems of aeroelasticity.

In order to demonstrate the application of the theories in the preceding sections, a steel chimney under the action of fluid flow is studied for 40 seconds. After this time, the fluid disappears. The chimney considered has circular crosssection and is supposed to clamped at the root and free and the tip. The first natural frequency is calculated as $0.977 \mathrm{~Hz}$. The diameter and the thickness of the structural shell vary from base to top as shown in table 1 .

The material properties of the steel are density $\rho=7850 \mathrm{~kg} / \mathrm{m}^{3}$, Young's modulos $E=2.1 \cdot 10^{11} \mathrm{~Pa}$, shear modulus $G=8.077 \cdot 10^{10} \mathrm{~Pa}$, and Poisson's ratio $\nu=0.3$. The fluid is air with dynamic viscosity $\mu=1.810^{-5} \mathrm{~N} \mathrm{~s} / \mathrm{m}^{2}$ and density $\rho=1.21 \mathrm{~kg} / \mathrm{m}^{3}$. Four different planes of fluid are used, corresponding to the positions at 30, 60, 80 and $90 \mathrm{~m}$ from the clamped edge. The velocity at inflow follows the $x$-direction and in the chimney tip is $20 \mathrm{~m} / \mathrm{s}$, fig. 2 .

Table 1: $\quad$ Data on the chimney.

\begin{tabular}{|c|c|c|}
\hline Height $[\mathrm{m}]$ & Diameter $[\mathrm{m}]$ & Thickness $[\mathrm{m}]$ \\
\hline $0-30$ & 5.20 & 0.022 \\
\hline $30-60$ & 4.10 & 0.019 \\
\hline $60-80$ & 3.20 & 0.015 \\
\hline $80-90$ & 2.20 & 0.012 \\
\hline
\end{tabular}




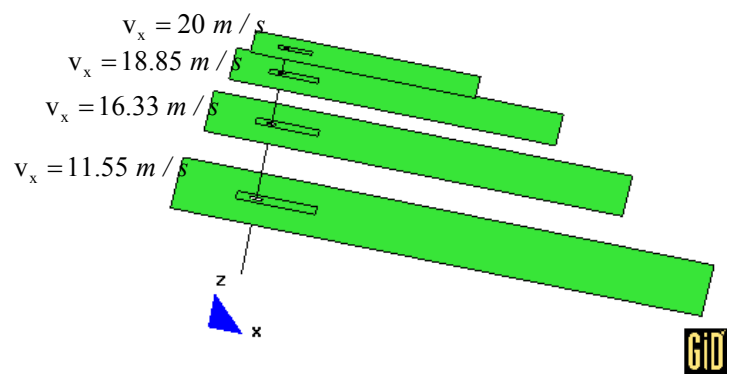

Figure 2: Chimney model the under wind action.

Since, four planes was used to simulate the fluid, the chimney was meshed with four beam elements. Structural damping (Rayleigh damping), proportional to the mass matrix, is assumed for the beam. To solve the coupled problem is necessary to solve the fluid and the structure separately; therefore a suitable time step has to be chosen, in our case 0.005 seconds.

In fig. 3 is shown the displacement time history in $y$-direction (cross-direction to wind direction) at the beam tip. As it is known, vortex-induced vibrations occur when vortices are shed alternately from opposite sides of the structure. This gives rise to a fluctuating load perpendicular to the wind direction. The frequency $n_{s}$ of this lateral load caused by vortex shedding is proportional to $U_{\infty} / d$. The factor of proportionality is called the Strouhal number St, eqn. (5)

$$
n_{s}=S t \frac{U_{\infty}}{d}
$$

where $U_{\infty}$ is the along-wind velocity and $d$ is the structure width perpendicular to the direction of the wind.

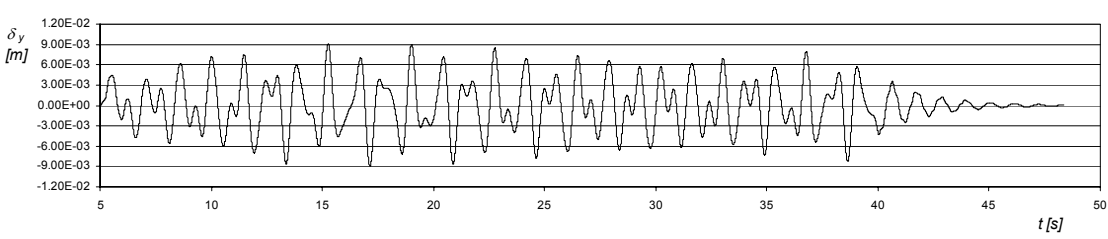

Figure 3: Displacement time history in $y$-direction.

According to eqn. (5), the frequencies of the lateral load are shown in table 2 for the different diameters of the chimney. Strouhal number $S t=0.2$ is assumed.

The corresponding Fourier analysis (Fast Fourier Transform) of displacement time history in $y$-direction, for time lower than 40 seconds, is given in fig. 4 . It is noticed that the chimney vibrates with frequencies near the calculated frequencies in theory (table 2). Indeed, the frequencies got numerically are 0.78 , 1.36 and $1.85 \mathrm{~Hz}$, against $0.79,1.17$ and $1.81 \mathrm{~Hz}$ values got from eqn. (5). In the same way, the FFT analysis shows a small peak near the $0.44 \mathrm{~Hz}$ value, indicated in table 2 . 
The FFT analysis of free vibration of the chimney is given in Figure 5. It is noticed that the chimney vibrates with its natural frequency.

Table 2: $\quad$ Frequencies of lateral load.

\begin{tabular}{|c|c|c|}
\hline Diameter, $d[\mathrm{~m}]$ & Velocity, $U_{\infty}[\mathrm{m} / \mathrm{s}]$ & Frequency, $n_{s}[\mathrm{~Hz}]$ \\
\hline 5.20 & 11.55 & 0.44 \\
\hline 4.10 & 16.33 & 0.79 \\
\hline 3.20 & 18.85 & 1.17 \\
\hline 2.20 & 20.00 & 1.81 \\
\hline
\end{tabular}

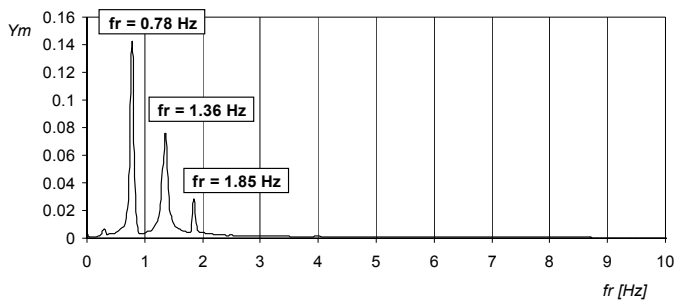

Figure 4: $\quad$ FFT analysis for time lower than $40 \mathrm{~s}$.

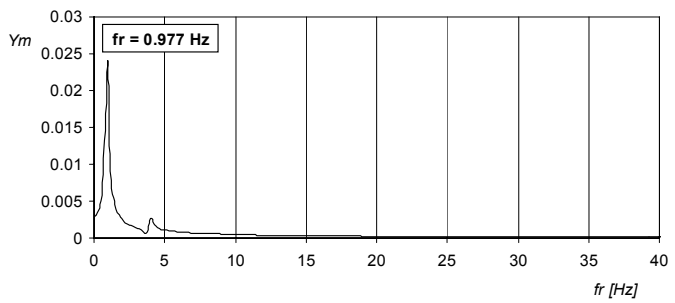

Figure 5: $\quad$ FFT analysis of free vibration.

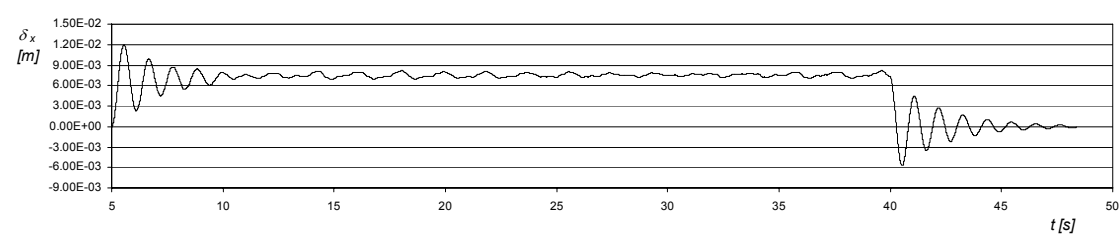

Figure 6: Displacement time history in $\mathrm{x}$-direction.

In fig. 6 is shown the displacement time history in $x$-direction at the beam tip. Initially, the chimney vibrates with bigger amplitude (transitory period) until it reaches stationary phase where it vibrates with lower amplitude around the new equilibrium position. When time is $40 \mathrm{~s}$, the fluid disappears and the chimney tends to recover the initial equilibrium position and vibrates with its natural frequency. The corresponding Fourier analysis (FFT) of displacement time history in $x$-direction, for time lower than $40 \mathrm{~s}$, is given in fig. 7. 


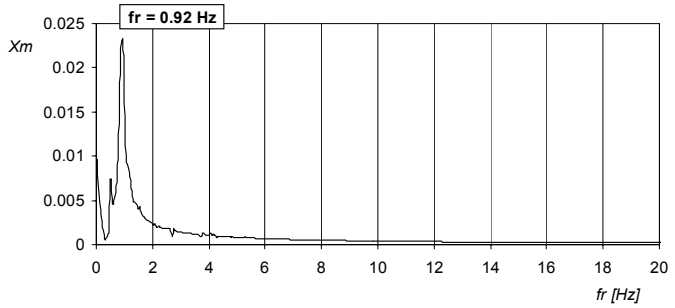

Figure 7: $\quad$ FFT analysis for time lower than 40 seconds.

\section{Conclusions}

A one-dimensional structural model applicable to thin-walled composite beams, which can have either an open or closed profile with either a single- or multiple-cell section, has been developed. The model includes the influence of the thickness of the shell wall and considers restrained torsion and secondary warping. Simple isotropic materials can be taken in account naturally.

On the other hand, current work focuses a FSI model which is appropriate for the simulation of slender beam structures subjected to wind load. The phenomenon of the flutter of a slender beam structure under the wind action is studied. In general, the predictions of the present analysis are in good agreement with theoretical results.

Finally, it has been possible to study successfully the free response of a slender beam structure when the action of the wind disappears. This opens the possibility of achieving simulations for structural control of the beam through the incorporation of control systems for the suppression of vibrations.

\section{Acknowledgement}

This work was supported by the Department of Science and Technology across the BIA2003-09078-C02-02 Project of the National Plan of I+D+I (2000-2003).

\section{References}

[1] Park, K. C. and Felippa, C., Synthesis tools for structural dynamics and partitioned analysis of coupled systems, www.colorado.edu/engineering /CAS/courses.d/FSI.d/NATO-ARW.Felippa.paper.rev.pdf.

[2] Felippa, C., FSI course: chapters 1-11, www.colorado.edu/engineering/ CAS/courses.d/FSI.d/FSI.Ch01.d/FSI.Ch01.pdf, 2004.

[3] Larroutorou, B., Piperno, S. and Farhat, C., Partitioned procedures for the transient solution of coupled aeroelastic problems. Part 1: Model problem, theory and two dimensional applications, Computer Methods in Applied Mechanics and Engineering, 124:79-112, 1995. 
[4] Farhat, C. and Piperno, S., Partitioned procedures for the transient solution of coupled aeroelastic problems. Part 2: Energy transfer analysis and three dimensional applications, Computer Methods in Applied Mechanics and Engineering, 190:3147-3170, 1995.

[5] Brown, G., Farhat, C. and Geuzaine, P., Application of a three-field nonlinear fluid-structure formulation to the prediction of the aeroelastic parameters of an f-16 fighter, Computers and Fluids, 32:3-29, 2003.

[6] Del Pin, F., Oñate, E., Idelsohn, S. R. and Aubry, R., The particle finite element method. An overview, International Journal of Computational Methods, 1:267-307, 2004.

[7] Hubner, B., Walhorn, E., Kolke, A. and Dinkler, D. ,Fluid - structure coupling within a monolithic model involving free surface flows, Computers \& Structures, Volume 83, Issues 25-26, pp. 2100-2111, 2005.

[8] Dinkler, D., Hubner, B. and Walhorn, E., A monolithic approach to fluidstructure interaction using space-time finite elements, CMAME, 2003.

[9] Gluck, M., Halfmann, A., Rank, E. et al, A partitioned solution approach for the fluid-structure interaction of wind and thin walled structures, Technical report, TU Munchen, Universitat Erlangen-Nurnberg, 2000.

[10] Halfmann, A., Rank, E., Scholz, D. et al, Fluid-structure interaction in civil engineering, In Second MIT conference on Computational Fluid And Solid Mechanics, 2003.

[11] Foces, A., Pereda, J., Cacho M. and Vasallo, A., Un modelo monodimensional para el análisis de barras de pared delgada de material compuesto, Avances en Mecánica de Medios Continuos, Servicio de Publicaciones de la Universidad de Valladolid, 2006.

[12] Rossi, R., Light Weight Structures: Structural Analysis and Coupling Issues, Ph. D. dissertation, Università di Bologna, 2005.

[13] Rossi, R., Lazzari, M., and Vitaliani, R., Wind field generation for structural engineering purposes, International Journal of Numerical Methods in Engineering, Vol 61, pp. 738-763, 2004.

[14] Lazzari M., Time domain modelling of aeroelastic bridge decks: a comparative study and an application, International Journal of Numerical Methods in Engineering, No. 62, 2005.

[15] Codina, R., Pressure Stability in Fractional Step Finite Elements Methods for Incompressible Flows, Journal of Computational Physics, 2001.

[16] Badia, S., Fractional step methods for incompressible flows, Fluidstructure interaction, Ph. D. dissertation, Universidad Politécnica de Cataluña, 2006.

[17] Codina, R., Stabilized finite element approximation of transient incompressible flows using orthogonal subscales, Computer Methods in Applied Mechanics and Engineering, 191, 4295-4321. 